\title{
Flows and Shocks in Variable Fluxes of Seyfert galaxies
}

\author{
I.I. Pronik \\ Crimean Astrophysical Observatory, p. Nauchny, Crimea, Ukraine
}

\begin{abstract}
Data collected from the optical through infrared to the radio show that the Seyfert galaxies NGC 1275, NGC 3227, NGC 4151, NGC 5548 and NGC 7469 exhibit signs of flows or jets. The characteristics of night-to-night variations of Balmer line profiles and/or intranight continuum variations were obtained and investigated at the Crimean Astrophysical Observatory. They permit to suppose, that short term variability of the Balmer lines and intranight continuum variations can be produced in short lived shocks as a result of strong energy output in the form of gas flows or jets.
\end{abstract}

\section{Spectral observations}

53 spectra for NGC 3227 and 23 spectra for NGC 7469 in the spectral region $3700-7300 \AA$ were obtained with 6-m telescope on January 12-15, 1977 during the maximum and minimum of the nucleus brightness, respectively. Equivalent widths $\left(\mathrm{EW}_{\lambda}\right)$ and profiles of the emission lines $\mathrm{H}_{\gamma}, \mathrm{H}_{\beta},\left(\mathrm{H}_{\alpha}+[\mathrm{NII}]\right)$ were used for short-term variability investigation.

The profiles of the Balmer lines $\mathrm{H}_{\gamma}$ and $\mathrm{H}_{\beta}$ in the spectra of NGC 3227 evolved from January 12 to 14 . At first the increasing of blue component of the $\mathrm{H}_{\beta}$ profile core was registered. Then relative intensities of other emission components and peak of the line profile increased with the decreasing of EW: FWHM increased by factor $\sim 2$ with the reduction of $\mathrm{EW}$ by factor $\sim 1.5$. FWHM in velocity units increased during 3 days by thousands $\mathrm{km} \mathrm{s}^{-1}$ up to $4500 \mathrm{~km} \mathrm{~s}^{-1}$ in the $\mathrm{H}_{\beta}$ and up to $5700 \mathrm{~km} \mathrm{~s}^{-1}$ in the $\mathrm{H}_{\gamma}$ line profiles.

The profiles of the Balmer lines in the NGC 7469 spectrum exhibited evolution from 13 to 15 January. Character of variations is the same as that of the Balmer lines in NGC 3227, but in opposite direction. We suppose that nightto-night emission line variability of both nuclei was caused by three day flare in broad line region (BLR).

Emission of night-to-night broad variable hydrogen lines has inverse Balmer decrement. Fitting of the observational data to the grid of theoretical models by Gershberg and Shnoll prompts to suppose that gas emitting variable Balmer lines is ionized and excited by collisional process. Electron temperature of flare gas is $\mathrm{T}_{e} \sim 25000 \mathrm{~K}$ and electron concentration $\mathrm{n}_{e} \sim\left(10^{12}-10^{14}\right) \mathrm{cm}^{-3}$. Supposition on collisional process in flares is supported also by the fact that the broadening of the lines during the flare is accompanied by decreasing of $\mathrm{EW}$ of the emission lines. 
We suppose that in BLR of the NGC 3227 and NGC 7469 there are two independent regions of activity. Dimension of large BLR is $\sim 4.5 \times 10^{16} \mathrm{~cm}$. Most characteristics of optical emission spectrum of large BLR can be understood on the basis of photoionization models by Osterbrock et al. using $n_{e} \sim 10^{10}-10^{11}$ $\mathrm{cm}^{-3}$. Large BLR contains flows and streams of dozen years old. Inside these flows there are small BLRs which are observed during short lived flares. Ionization and excitation of small variable BLR with $\mathrm{n}_{e} \sim 10^{12}-10^{14} \mathrm{~cm}^{-3}$ are caused by collisional processes. Velocities of emitting clouds increased by thousands $\mathrm{km} \mathrm{s}^{-1}$. Dimension of small BLR equals $\sim 3$ light-day $\left(\sim 8 \times 10^{15} \mathrm{~cm}\right)$. Short time-flares was not connected with the general brightness of the galaxy nuclei since the flare in the NGC 7469 was observed during minimum brightness while the flare in NGC 3227 - during maximum brightness of the nucleus.

\section{Photoelectrical observations}

Photoelectrical observations were made of the Seyfert galaxies NGC 1275, NGC 4151, NGC 5548 and NGC 7469 with the 1.25-m telescope of the Crimean Astrophysical Observatory beginning the 1982. Since 1989 observations were performed in the standard Johnson $U B V R I$ photometric system simultaneously during $5-8$ years.

Ratios of Standard Deviations (SD) of the variable flux to the nightly average flux $\mathrm{F}$ for each night- $\mathrm{SD} / \mathrm{F}$, and the parameters of Structure Functions (SF)-b, were used for discussion of the variability character. Signs of different characteristics of intranight and infranight variations were revealed:

1. $\mathrm{SD} / \mathrm{F}$ of intranight variations are independent on the level of nightly averaged values of the flux.

2. The intranight variations as a rule show higher values of SF parameters $b$ $=\mathrm{d} \log (\mathrm{SF}) / \mathrm{d} \log (\mathrm{dt})$ than those of infranight variations by factor 2 and more.

3. Signs of two independent sources of intranight variations and only one source of infranight variations were obtained for the NGC 1275, NGC 5548 and NGC 7469. One of the sources of intranight variations is more powerful in ultraviolet and another one - in infrared.

Obtained photoelectrical data evidenced in favor of supposition that intranight and extranight variations of the Seyfert galaxy nuclei NGC 1275, NGC 4151, NGC 5548 and NGC 7469 are caused by different sources with the independent activity. SF parameter "b" can be interpreted as a characteristics of energy output rate. Therefore obtained data evidenced that the rate of energy output of intranight variations is essentially higher than that of infranight ones.

\section{Conclusions}

More suitable understanding of the observations can be in framework of a model of short lived shocks in gas flows or jets. Rates of energy output inside shocks are stronger than those of long time variations. We suppose that shocks acted mainly at the boundary of the accretion disk because double-peaked cores of the line profiles varied during the flares. Shock activity causing few-day and intranight flares is independent on variations of general brightness of the nuclei. 